\section{PTU-9 POST-COLONOSCOPY COLORECTAL CANCER: A 5-YEAR ANALYSIS AND MEASURES IMPLEMENTED AT A DISTRICT GENERAL HOSPITAL}

Nick Constantinou*, Jodie Chalmers, Thomas Marjot, Sue Cullen, Emily Johns, Sujata Biswas. Buckinghamshire Healthcare NHS Trust, UK

\subsection{6/gutjnl-2021-BSG.82}

Introduction Post-colonoscopy colorectal cancer (PCCRC) is the diagnosis of cancer up to 3 years after a colonoscopy at which no cancer was found. It is a measure of endoscopy quality assurance and PCCRC rates are expected as part of both JAG assessment and the Getting It Right First Time programme. The WEO (World Endoscopy Organisation) has published guidelines for calculation and categorisation of PCCRCs. We analysed local PCCRC rates and established measures to improve these over time.

Methods Retrospective analysis of colonoscopies performed from 1st January 2014 to 31st December 31st 2016 was done. The endoscopy and pathology databases and lower GI cancer MDT lists were utilised. PCCRCs were determined up to 3 years after initial investigation, and rates calculated using WEO guidelines.

Results 11,268 colonoscopies were performed between 2014 and 2016. Annual PCCRC rates reduced incrementally each year, from $10.1 \%$ in 2014 , to $4.5 \%$ in 2015 , to $3.5 \%$ in 2016 (Table 1). The majority of cases were due to a missed lesion at a prior examination which was thought to be adequate. In cases of uncertainty $(n=7)$ a Root Cause Analysis (RCA) was performed.

All PCCRC cases were fed back to the responsible endoscopist and audit data were presented at endoscopy user group meetings. An incident form was completed within 48 hours of reporting, and if the case was deemed to be potentially preventable, a Serious Incident report was filed. These reports and RCAs were discussed at Clinical Governance meetings. Patients were informed using a standard Duty of Candour letter, and invited to an individual meeting to discuss lessons learnt and actions taken.

\begin{tabular}{llll}
$\begin{array}{l}\text { Abstract PTU-9 Table 1 } \\
2014 \text { to } 2016\end{array}$ & & & \\
\hline & 2014 & 2015 & 2016 \\
\hline No. colonoscopies & 3298 & 3556 & 4414 \\
No. patients with colonoscopy and CRC & 149 & 132 & 141 \\
No. detected cancers (<6m after colonoscopy) & 132 & 126 & 136 \\
No. PCCRC (6-36m after colonoscopy) & 15 & 6 & 5 \\
PCCRC 3 yr rate & $10.1 \%$ & $4.5 \%$ & $3.5 \%$ \\
& & & \\
Categorisation of PCCRC & & & \\
Possible missed lesion, prior examination & $27 \%$ & $33 \%$ & $60 \%$ \\
adequate & $(4 / 15)$ & $(2 / 6)$ & $(3 / 5)$ \\
Possible missed lesion, prior examination & $13 \%$ & $0 \%$ & $20 \%$ \\
inadequate & $(2 / 15)$ & $(0 / 6)$ & $(1 / 5)$ \\
Detected lesion, not resected & $7 \%$ & $33 \%$ & $0 \%$ \\
& $(1 / 15)$ & $(2 / 6)$ & $(0 / 5)$ \\
Likely incomplete resection & $20 \%$ & $17 \%$ & $0 \%$ \\
& $(3 / 15)$ & $(1 / 6)$ & $(0 / 5)$ \\
Uncertainty requiring RCA & $33 \%$ & $17 \%$ & $20 \%$ \\
& $(5 / 15)$ & $(1 / 6)$ & $(1 / 5)$ \\
\hline
\end{tabular}

Conclusions This retrospective audit demonstrates that our rates are consistent with the national average, and that incremental improvements are possible. The gold standard 3-year PCCRC rate of $2 \%$ suggested in a recent paper $^{1}$ should be achievable with the correct measures. We recommend regular audits, standardised documents to report incidents and to inform patients, and evaluation at departmental meetings.

\section{REFERENCE}

1. Anderson R, Burr NE, Valori R. Causes of Post-Colonoscopy Colorectal Cancers Based on World Endoscopy Organization System of Analysis. Gastroenterology 2020;158(5):1287-1299.

\section{PTU-10 BOTULINUM TOXIN INJECTION IN THE MANAGEMENT OF TYPE II SPHINCTER OF ODDI DYSFUNCTION}

Shyam Menon, Ray Mathew. The Royal Wolverhampton Nhs Trust, Wolverhampton, UK

\subsection{6/gutjnl-2021-BSG.83}

Introduction Type II sphincter of Oddi dysfunction (SOD) or functional biliary sphincter disorder (FBSD) is treated by endoscopic biliary sphincterotomy. However, a proportion of patients continue to experience attacks of severe pancreaticobiliary pain necessitating hospital admissions for management of acute pain. Intrasphincteric Botulinum toxin (Botox) injection to the sphincter of Oddi has been demonstrated previously to alleviate pain in FBSD and to identify patients who may respond to an endoscopic biliary sphincterotomy (ES). Botox has been demonstrated to be useful in managing pain in patients with Type III SOD. We have been using Botox in symptomatic patients with FBSD post ES and report our experience with this strategy.

Methods A retrospective review of case notes over a 5-year period (2014-2019) was performed. All patients underwent extensive investigations including blood tests, gastroscopy, trans-abdominal ultrasonography, cross-sectional imaging with $\mathrm{MRCP} / \mathrm{CT}$ and EUS, and Morphine TBIDA scans. The diagnosis of Type II SOD or FBSD was made in post cholecystectomy patients with abdominal pain identical to their precholecystectomy pain and either a dilated bile duct or anormal liver function tests (ALT or ALP x 1.5 times upper limit of normal) in line with the modified Milwaukee criteria and/or a positive Morphine TBIDA scan. All patients underwent ES and were followed up in an outpatient setting. Patients who had ongoing symptoms of severe pancreaticobiliary pain following ES underwent Botox injection around the ampullary area. Further Botox injections were applied depending on duration of response. The efficacy of ampullary botulinum toxin injection on pain was recorded at post-procedure outpatient review using a nominal pain scale. Opioid analgesia and frequency of hospital admissions were noted, in addition to neuromodulatory medication initiated at the time of endoscopy or at subsequent outpatient review.

Results 15 female patients with FBSD who underwent ES and who had ongoing pancreaticobiliary pain underwent a mean of 3 (1-7) Botox injections over a mean period of 15 (7-4) months. The median dose of botulinum toxin used was 200 (100-600) units. 14/15 (93\%) patients reported a significant improvement in pain on post procedure review with 13/15 $(87 \%)$ of patients managing to discontinue opioid medication post procedure. $9 / 14(64 \%)$ had a complete response to pain and $5 / 14(36 \%)$ had a partial response. 1 patient had no response to Botox injection and continued on opioid therapy. 